Bull. Korean Math. Soc. 50 (2013), No. 2, pp. 675-685

http://dx.doi.org/10.4134/BKMS.2013.50.2.675

\title{
UNIFORMLY BOUNDED COMPOSITION OPERATORS ON A BANACH SPACE OF BOUNDED WIENER-YOUNG VARIATION FUNCTIONS
}

\author{
Dorota G£azowska, José Atilio Guerrero, Janusz Matkowski, \\ ANd Nelson Merentes
}

\begin{abstract}
We prove, under some general assumptions, that a generator of any uniformly bounded Nemytskij operator, mapping a subset of space of functions of bounded variation in the sense of Wiener-Young into another space of this type, must be an affine function with respect to the second variable.
\end{abstract}

\section{Introduction}

Let $I, X, Y$ be arbitrary nonempty sets. For a given function $h: I \times X \longrightarrow Y$, the mapping $H: X^{I} \longrightarrow Y^{I}$ defined by

$$
(H f)(t)=h(t, f(t)), \quad f \in X^{I}(t \in I),
$$

is called the Nemytskij composition operator of generator $h$ (here $X^{I}$ stands for the family of all functions $f: I \longrightarrow X)$. In the sequel we assume that $I$ is a real interval.

The composition operators play important role in the theory of differential, integral and functional equations. In some function spaces any locally defined operator (or operator with memory) must be a composition operator (cf. [5, $16,17,18,19,20])$.

It is known that if a composition operator maps the Banach space $\operatorname{Lip}(I, \mathbb{R})$ into itself and is globally Lipschitzian with respect to the Lip-norm, then its generator $h$ must be of the form

$$
h(t, x)=a(t) x+b(t), \quad t \in I, x \in \mathbb{R}
$$

Received January 17, 2012; Revised October 11, 2012.

2010 Mathematics Subject Classification. Primary 47B33, 26B30, 26B40; Secondary 39B22.

Key words and phrases. $\varphi$-variation in the sense of Wiener, uniformly bounded operator, regularization, composition operator, Jensen equation.

This research has been partly supported by Central Bank of Venezuela. We also want to give thanks to the library staff of B.C.V. for compiling the references.

The second author was partially funded by the Decanato de Investigación-Universidad Nacional Experimental del Táchira-Venezuela. 
for some $a, b \in \operatorname{Lip}(I, \mathbb{R})$ (cf. [8]). This result has been extended to some other function Banach spaces (cf. [1] and the references therein). Then it turned out that the Lipschitz continuity of the composition operators can be replaced by its uniform continuity [10].

Recently a new essential improvements has been done in [10], where it is shown that the generator of any uniformly bounded composition operator (Definition 3.5) mapping the Banach space of Lipschitz functions into itself (or into another space of that type) must be of the above form. Let us note that, every bounded operator is uniformly bounded.

Let $X$ and $Y$ be real Banach spaces, and $C \subset X$ a convex set. Denote by $\left(B V_{\varphi}(I, X),\|\cdot\|_{\varphi}\right)$ the Banach space of functions $f: I \rightarrow X$ which are of bounded $\varphi$-variation in the sense of Wiener [15], (or more, generally in the sense of Young [21]) where the norm $\|\cdot\|_{\varphi}$ is defined with the aid of LuxemburgNakano-Orlicz seminorm $[6,13,14]$. Assume that $H$ maps the set of functions $f \in B V_{\varphi}(I, X)$ such that $f(I) \subset C$ into $B V_{\psi}(I, Y)$. The main result of the present paper (Theorem 3.3) reads as follows: if the operator $H$ is uniformly bounded, then (2) holds. As a very special case we obtain the result of [3], where $H$ is assumed to be uniformly continuous.

This result implies that, in general, the Banach fixed point theorem (or any of its generalizations) can be applied to determine the solutions $f \in B V_{\varphi}(I, X)$ of the functional equation

$$
f(t)=h(t, f(\alpha(t))), \quad t \in I,
$$

where $h: I \times X \rightarrow X$ and $\alpha: I \rightarrow I$ are given, only if this equation has the form

$$
f(t)=a(t) f(\alpha(t))+b(t), \quad t \in I,
$$

i.e., only if the equation is linear.

\section{Preliminaries}

In this section we present some definitions and preliminary results related to the functions of bounded $\varphi$-variation in the sense of Wiener.

Let $\mathcal{F}$ be the set of all convex functions $\varphi:[0, \infty) \longrightarrow[0, \infty)$ such that $\varphi\left(0^{+}\right)=\varphi(0)=0, \varphi(t)>0$ for $t>0$ and $\lim _{t \rightarrow \infty} \varphi(t)=\infty$.

Remark 1.1. If $\varphi \in \mathcal{F}$, then $\varphi$ is continuous and strictly increasing. Indeed, the convexity of $\varphi$ and $\varphi(0)=0$ imply the continuity of $\varphi$. Suppose that $\varphi\left(t_{1}\right) \geq \varphi\left(t_{2}\right)$ for some $0<t_{1}<t_{2}$. Then

$$
\frac{\varphi\left(t_{1}\right)-\varphi(0)}{t_{1}-0}=\frac{\varphi\left(t_{1}\right)}{t_{1}}>\frac{\varphi\left(t_{2}\right)}{t_{2}}=\frac{\varphi\left(t_{2}\right)-\varphi(0)}{t_{2}-0},
$$

contradicting the convexity of $\varphi$.

Definition 1.2. Let $\varphi \in \mathcal{F}$ and $(X,|\cdot|)$ be a real normed space, and $I \subset \mathbb{R}$ be an interval. A function $f \in X^{I}$ is of bounded $\varphi$-variation in the sense of 
Wiener-Young in $I$, if

$$
v_{\varphi}(f)=v_{\varphi}(f, I):=\sup _{\xi} \sum_{i=1}^{m} \varphi\left(\left|f\left(t_{i}\right)-f\left(t_{i-1}\right)\right|\right)<\infty
$$

where the supremum is taken over all finite and increasing sequences $\xi=$ $\left(t_{i}\right)_{i=0}^{m}, t_{i} \in I, m \in \mathbb{N}$.

For $\varphi(t)=t^{p}(t \geq 0, p \geq 1)$, condition (3) coincides with the classical concept of variation in the sense of Jordan whenever $p=1$, and in the sense of Wiener [15] if $p>1$. The general Definition 1.2 was introduced by Young [21].

Denote the set of all bounded $\varphi$-variation functions $f \in X^{I}$ in the sense of Wiener by $V_{\varphi}(I, X)$. This is a symmetric and convex set but it is not necessarily a linear space, Musielak and Orlicz $[12]$ proved that $V_{\varphi}(I, \mathbb{R})$ is a vector space if and only if $\varphi$ satisfies the $\Delta_{2}$ condition.

Denote by $B V_{\varphi}(I, X)$ the linear space of all functions $f \in X^{I}$ such that $v_{\varphi}(\lambda f)<\infty$ for some constant $\lambda>0$. In this space the function $\|\cdot\|_{\varphi}$ defined by

$$
\|f\|_{\varphi}:=\left|f\left(t_{0}\right)\right|+p_{\varphi}(f), \quad f \in B V_{\varphi}(I, X),
$$

where $t_{0} \in I$ is fixed, and

$$
p_{\varphi}(f):=p_{\varphi}(f, I)=\inf \left\{\epsilon>0: v_{\varphi}(f / \epsilon) \leq 1\right\}, f \in B V_{\varphi}(I, X)
$$

is a norm (see for instance [12]).

Maligranda and Orlicz [7], proved that the space $\left(B V_{\varphi}(I, \mathbb{R}),\|\cdot\|_{\varphi}\right)$ is a Banach algebra. The functional $p_{\varphi}(\cdot)$ defined by $(4)$ is called the LuxemburgNakano-Orlicz seminorm $[6,13,14]$.

In what follows we shall need the following lemmas.

Lemma 1.3 (Chistyakov [2, Lemma 1]). For $f \in B V_{\varphi}(I, X)$ and $\lambda>0$, we have:

(a) $p_{\varphi}(f) \leq \lambda$ if and only if $v_{\varphi}(f / \lambda) \leq 1$;

(b) if $v_{\varphi}(f / \lambda)=1$, then $p_{\varphi}(f)=\lambda$.

Let $B V_{\varphi}^{-}\left(I^{-}, X\right)$ denote the subset of $B V_{\varphi}(I, X)$ consisting of those functions which are left continuous on $I^{-}:=I \backslash\{\inf I\}$, and let $B V_{\varphi}^{+}\left(I^{+}, X\right)$ denote the subset of $B V_{\varphi}(I, X)$ that consists of those functions which are right continuous on $I^{+}:=I \backslash\{\sup I\}$.

If $(X,|\cdot|)$ is a Banach space and $f \in B V_{\varphi}(I, X)$, then

$$
f^{-}(t):=\lim _{s \uparrow t} f(s), \quad t \in I^{-} ; \quad \text { and } \quad f^{+}(t):=\lim _{s \downarrow t} f(s), \quad t \in I^{+},
$$

exist and they are called the left regularization and right regularization of $f$ (cf. [11]).

Lemma 1.4 (Chistyakov [2, Lemma 6]). If $X$ is a Banach space and $f \in$ $B V_{\varphi}(I, X)$, then $f^{-} \in B V_{\varphi}^{-}\left(I^{-}, X\right)$ and $f^{+} \in B V_{\varphi}^{+}\left(I^{+}, X\right)$. 


\section{An auxiliary result and its consequences}

For a set $C \subset X$, let us define

$$
B V_{\varphi}(I, C):=\left\{f \in B V_{\varphi}(I, X) \mid f(I) \subset C\right\} .
$$

For the real linear space $X, Y$ denote by $\mathcal{A}(X, Y)$ the set of all additive mappings from $X$ into $Y$ and, if $X, Y$ are normed spaces by $\mathcal{L}(X, Y)$, the set of all linear and continuous mappings from $X$ into $Y$.

Following an idea of [3] and [9], we prove:

Theorem 2.1. Let $I \subset \mathbb{R}$ be an arbitrary interval, $\varphi, \psi \in \mathcal{F},\left(X,|\cdot|_{X}\right)$ and $\left(Y,|\cdot|_{Y}\right)$ be real Banach spaces, $C \subset X$ a convex set such that int $C \neq \emptyset$. Suppose that the function $h: I \times C \longrightarrow Y$ is continuous with respect to the second variable. If the composition operator $H: C^{I} \longrightarrow Y^{I}$ generated by $h$, maps $B V_{\varphi}(I, C)$ into $B V_{\psi}(I, Y)$ and satisfies the inequality

$$
\left\|H\left(f_{1}\right)-H\left(f_{2}\right)\right\|_{\psi} \leq \gamma\left(\left\|f_{1}-f_{2}\right\|_{\varphi}\right), \quad f_{1}, f_{2} \in B V_{\varphi}(I, C),
$$

for some function $\gamma:[0, \infty) \longrightarrow[0, \infty)$, then

(i) there exist $a^{-}: I^{-} \longrightarrow \mathcal{L}(X, Y)$ and $b^{-} \in B V_{\psi}\left(I^{-}, Y\right)$ such that

$$
h^{-}(t, x)=a^{-}(t) x+b^{-}(t), \quad t \in I^{-}, x \in C,
$$

where $h^{-}: I^{-} \times C \longrightarrow Y$ is the left regularization of $h$;

(ii) there exist $a^{+}: I^{+} \longrightarrow \mathcal{L}(X, Y)$ and $b^{+} \in B V_{\psi}\left(I^{+}, Y\right)$ such that

$$
h^{+}(t, x)=a^{+}(t) x+b^{+}(t), \quad t \in I^{+}, x \in C,
$$

where $h^{+}: I^{+} \times C \longrightarrow Y$ is the right regularization of $h$.

Proof. For every $x \in C$, the constant function $f(t)=x(t \in I)$ belongs to $B V_{\varphi}(I, C)$. Since $H$ maps $B V_{\varphi}(I, C)$ into $B V_{\psi}(I, Y)$, it follows that the function $I \ni t \longmapsto h(t, x)$ belongs to $B V_{\psi}(I, Y)$. The existence of the left regularization $h^{-}$of $h$ follows from Lemma 1.4 and the completeness of $\left(Y,|\cdot|_{Y}\right)$.

From (5), by the definition of the norm $\|\cdot\|_{\psi}$, we obtain

$$
p_{\psi}\left(H\left(f_{1}\right)-H\left(f_{2}\right)\right) \leq\left\|H\left(f_{1}\right)-H\left(f_{2}\right)\right\|_{\psi} \quad \text { for } \quad f_{1}, f_{2} \in B V_{\varphi}(I, C) .
$$

From (5), (6) and Lemma 1.3(a), if $\gamma\left(\left\|f_{1}-f_{2}\right\|_{\varphi}\right)>0$, then

$$
v_{\psi}\left(\frac{H\left(f_{1}\right)-H\left(f_{2}\right)}{\gamma\left(\left\|f_{1}-f_{2}\right\|_{\varphi}\right)}\right) \leq 1
$$

Therefore, for any $s_{1}<t_{1}<s_{2}<t_{2}<\cdots<s_{m}<t_{m}, s_{i}, t_{i} \in I, i \in$ $\{1,2, \ldots, m\}, m \in \mathbb{N}$, the definitions of the operator $H$, the functional $v_{\psi}$ and (7) imply

(8) $\sum_{i=1}^{m} \psi\left(\frac{\left|h\left(t_{i}, f_{1}\left(t_{i}\right)\right)-h\left(t_{i}, f_{2}\left(t_{i}\right)\right)-h\left(s_{i}, f_{1}\left(s_{i}\right)\right)+h\left(s_{i}, f_{2}\left(s_{i}\right)\right)\right|}{\gamma\left(\left\|f_{1}-f_{2}\right\|_{\varphi}\right)}\right) \leq 1$. 
For $s^{\prime}, t^{\prime} \in \mathbb{R}, s^{\prime}<t^{\prime}$, define the functions $\eta_{s^{\prime}, t^{\prime}}: \mathbb{R} \longrightarrow[0,1]$ by

$$
\eta_{s^{\prime}, t^{\prime}}(t):= \begin{cases}0 & \text { if } \quad t \leq s^{\prime} \\ \frac{t-s^{\prime}}{t^{\prime}-s^{\prime}} & \text { if } \quad s^{\prime} \leq t \leq t^{\prime} \\ 1 & \text { if } \quad t^{\prime} \leq t\end{cases}
$$

Let us fix $r \in I^{-}$. For arbitrary finite sequence inf $I<s_{1}<t_{1}<s_{2}<t_{2}<$ $\cdots<s_{m}<t_{m}<r$ and $x_{1}, x_{2} \in C, x_{1} \neq x_{2}$, the functions $f_{1}, f_{2}: I \longrightarrow X$ defined by

$$
f_{k}(t):=\frac{1}{2}\left[\eta_{s_{i}, t_{i}}(t)\left(x_{1}-x_{2}\right)+x_{k}+x_{2}\right], \quad t \in I, k=1,2,
$$

belong to the space $B V_{\varphi}(I, C)$. From (10) and (9), we have

$$
f_{1}(\cdot)-f_{2}(\cdot)=\frac{x_{1}-x_{2}}{2},
$$

whence, obviously,

$$
\left\|f_{1}-f_{2}\right\|_{\varphi}=\frac{\left|x_{1}-x_{2}\right|}{2}
$$

and, moreover

$$
f_{1}\left(t_{i}\right)=x_{1} ; \quad f_{2}\left(t_{i}\right)=\frac{x_{1}+x_{2}}{2} ; \quad f_{1}\left(s_{i}\right)=\frac{x_{1}+x_{2}}{2} ; \quad f_{2}\left(s_{i}\right)=x_{2} .
$$

Using (8), we hence get

$$
\text { (11) } \sum_{i=1}^{m} \psi\left(\frac{\left|h\left(t_{i}, x_{1}\right)-h\left(t_{i}, \frac{x_{1}+x_{2}}{2}\right)-h\left(s_{i}, \frac{x_{1}+x_{2}}{2}\right)+h\left(s_{i}, x_{2}\right)\right|}{\gamma\left(\frac{\left|x_{1}-x_{2}\right|}{2}\right)}\right) \leq 1 \text {. }
$$

From the continuity of $\psi$ and the definition of $h^{-}$, letting $s_{1} \uparrow r$ in (11), we obtain

$$
\sum_{i=1}^{m} \psi\left(\frac{\left|h^{-}\left(r, x_{1}\right)-h^{-}\left(r, \frac{x_{1}+x_{2}}{2}\right)-h^{-}\left(r, \frac{x_{1}+x_{2}}{2}\right)+h^{-}\left(r, x_{2}\right)\right|}{\gamma\left(\frac{\left|x_{1}-x_{2}\right|}{2}\right)}\right) \leq 1,
$$

that is

$$
\psi\left(\frac{\left|h^{-}\left(r, x_{1}\right)-2 h^{-}\left(r, \frac{x_{1}+x_{2}}{2}\right)+h^{-}\left(r, x_{2}\right)\right|}{\gamma\left(\frac{\left|x_{1}-x_{2}\right|}{2}\right)}\right) \leq \frac{1}{m} .
$$

Hence, since $m \in \mathbb{N}$ is arbitrary,

$$
\psi\left(\frac{\left|h^{-}\left(r, x_{1}\right)-2 h^{-}\left(r, \frac{x_{1}+x_{2}}{2}\right)+h^{-}\left(r, x_{2}\right)\right|}{\gamma\left(\frac{\left|x_{1}-x_{2}\right|}{2}\right)}\right)=0,
$$


and, as $\psi(z)=0$ only if $z=0$, we obtain

$$
\left|h^{-}\left(r, x_{1}\right)-2 h^{-}\left(r, \frac{x_{1}+x_{2}}{2}\right)+h^{-}\left(r, x_{2}\right)\right|=0 \text {. }
$$

Therefore

$$
h^{-}\left(r, \frac{x_{1}+x_{2}}{2}\right)=\frac{h^{-}\left(r, x_{1}\right)+h^{-}\left(r, x_{2}\right)}{2}, \quad r \in I^{-}, x_{1}, x_{2} \in C .
$$

In the sequel we apply the method presented in M. Kuczma [4, pp. 314-315] for functions of real variable. Let us fix $t \in I^{-}, x_{0} \in C$. Let $C_{0}:=C-x_{0}$ and

$$
g(x):=h^{-}\left(t, x+x_{0}\right)-h^{-}\left(t, x_{0}\right), \quad x \in C_{0} .
$$

From (13) and (12), for all $x_{1}, x_{2} \in C_{0}$, we get

$$
\begin{aligned}
g\left(\frac{x_{1}+x_{2}}{2}\right) & =h^{-}\left(t, \frac{x_{1}+x_{2}}{2}\right)-h^{-}\left(t, x_{0}\right) \\
& =\frac{h^{-}\left(t, x_{1}\right)+h^{-}\left(x_{2}\right)}{2}-h^{-}\left(t, x_{0}\right) \\
& =\frac{g\left(x_{1}\right)+g\left(x_{2}\right)}{2},
\end{aligned}
$$

which shows that the function $g$ is Jensen affine in $C_{0}$. Since $0 \in C_{0}$ and

$$
g(0)=h^{-}(t, 0)-h^{-}(t, 0)=0,
$$

by induction, we hence get that

$$
2^{k} g\left(\frac{x}{2^{k}}\right)=g(x), \quad x \in C_{0}, k \in \mathbb{N} .
$$

Denote by $\operatorname{span}\left(C_{0}\right)$ the set

$$
\operatorname{span}\left(C_{0}\right)=\left\{\sum_{i=1}^{n} \lambda_{i} x_{i} \mid \text { for some } n \in \mathbb{N}, \lambda_{i} \in \mathbb{R}, x_{i} \in C_{0}, i=1, \ldots, n\right\}
$$

Of course, for every $z \in \operatorname{span}\left(C_{0}\right)$ there is a positive integer $n=n(z)$ such that $2^{-n} z \in C_{0}$. Put

$$
G(z):=2^{n} g\left(\frac{z}{2^{n}}\right), \quad z \in \operatorname{span}\left(C_{0}\right), n=n(z) .
$$

Since the value $G(z)$ does not depend on the choice of $n=n(z)$, the function $G: \operatorname{span}\left(C_{0}\right) \longrightarrow Y$ is correctly defined. As for $z \in \operatorname{span}\left(C_{0}\right)$ one can choose $n(z)=1$, we have

$$
G(z)=g(z), \quad z \in C_{0} .
$$

Take arbitrary $z_{1}, z_{2} \in \operatorname{span}\left(C_{0}\right)$ and choose a positive $n$ such that $\frac{z_{1}}{2^{n}}, \frac{z_{2}}{2^{n}} \in$ $C_{0}$. By the convexity of $C_{0}$,

$$
\frac{\frac{z_{1}}{2^{n}}+\frac{z_{2}}{2^{n}}}{2}=\frac{z_{1}+z_{2}}{2^{n+1}} \in C_{0}
$$


Now, applying in turn the definition of $G$, Jensen affinity of $g$, and again the definition of $G$, we hence get, for arbitrary $z_{1}, z_{2} \in \operatorname{span}\left(C_{0}\right)$,

$$
\begin{aligned}
G\left(z_{1}+z_{2}\right) & =2^{n+1} g\left(\frac{z_{1}+z_{2}}{2^{n+1}}\right)=2^{n+1} g\left(\frac{\frac{z_{1}}{2^{n}}+\frac{z_{2}}{2^{n}}}{2}\right)=2^{n+1} \frac{g\left(\frac{z_{1}}{2^{n}}\right)+g\left(\frac{z_{2}}{2^{n}}\right)}{2} \\
& =2^{n} g\left(\frac{z_{1}}{2^{n}}\right)+2^{n} g\left(\frac{z_{2}}{2^{n}}\right)=G\left(z_{1}\right)+G\left(z_{2}\right),
\end{aligned}
$$

that is $G$ is additive in $\operatorname{span}\left(C_{0}\right)$. The continuity of $h^{-}(t, \cdot)$ implies the continuity of $G$. Consequently, being additive, $G$ must be linear, i.e., $G \in \mathcal{L}(X, Y)$. For $x \in C$, we have $x-x_{0} \in C_{0}$. Hence, from (13), we get

$$
\begin{aligned}
h^{-}(t, x) & =h^{-}\left(t,\left(x-x_{0}\right)+x_{0}\right)=g\left(x-x_{0}\right)+h^{-}\left(t, x_{0}\right) \\
& =G\left(x-x_{0}\right)+h^{-}\left(t, x_{0}\right) \\
& =G(x)-G\left(x_{0}\right)+h^{-}\left(t, x_{0}\right)
\end{aligned}
$$

whence, setting $a^{-}(t):=G$ and $b^{-}(t):=h^{-}\left(t, x_{0}\right)-G\left(x_{0}\right)$, we obtain

$$
h^{-}(t, x)=a^{-}(t) x+b^{-}(t), \quad t \in I^{-}, x \in C,
$$

and $b^{-} \in B V_{\psi}\left(I^{-}, Y\right)$.

The proof of (ii) is analogous.

Remark 2.2. If the function $\gamma:[0, \infty) \longrightarrow[0, \infty)$ is right continuous at 0 and $\gamma(0)=0$, then the assumption of the continuity of $h$ with respect to the second variable can be omitted.

Proof. For arbitrary fixed $x, \bar{x} \in C, x \neq \bar{x}$, define the pair of constant functions $f_{1}, f_{2}: I \longrightarrow C$ by

$$
f_{1}(t)=x, \quad f_{2}(t)=\bar{x}, \quad t \in I .
$$

Then of course $f, g \in B V_{\varphi}(I, C)$ and

$$
\left\|f_{1}-f_{2}\right\|_{\varphi}=|x-\bar{x}| \text {. }
$$

Moreover, by assumption, $H\left(f_{1}\right)=h(\cdot, x)$ and $H\left(f_{2}\right)=h(\cdot, \bar{x})$ belong to $B V_{\psi}(I, Y)$. From the definitions of the norm $\|\cdot\|_{\psi}$ and (5), we obtain

$$
\left|\left(H\left(f_{1}\right)-H\left(f_{2}\right)\right)\left(t_{0}\right)\right|+p_{\psi}\left(H\left(f_{1}\right)-H\left(f_{2}\right)\right) \leq \gamma(|x-\bar{x}|) \text { for some } t_{0} \in I,
$$

therefore

and

$$
\left|\left(H\left(f_{1}\right)-H\left(f_{2}\right)\right)\left(t_{0}\right)\right| \leq \gamma(|x-\bar{x}|)
$$

By (5), (6) and Lemma 1.3(a)

$$
p_{\psi}\left(H\left(f_{1}\right)-H\left(f_{2}\right)\right) \leq \gamma(|x-\bar{x}|)
$$

$$
v_{\psi}\left(\frac{H\left(f_{1}\right)-H\left(f_{2}\right)}{\gamma(|x-\bar{x}|)}\right) \leq 1,
$$

whence

$$
\psi\left(\frac{\left|\left(H\left(f_{1}\right)-H\left(f_{2}\right)\right)(t)-\left(H\left(f_{1}\right)-H\left(f_{2}\right)\right)\left(t_{0}\right)\right|}{\gamma(|x-\bar{x}|)}\right) \leq 1
$$


i.e.,

$$
\left|h(t, x)-h(t, \bar{x})-h\left(t_{0}, x\right)+h\left(t_{0}, \bar{x}\right)\right| \leq \psi^{-1}(1) \gamma(|x-\bar{x}|) .
$$

Thus

$$
\begin{aligned}
|h(t, x)-h(t, \bar{x})| & \leq\left|h(t, x)-h(t, \bar{x})-h\left(t_{0}, x\right)+h\left(t_{0}, \bar{x}\right)\right|+\left|h\left(t_{0}, x\right)-h\left(t_{0}, \bar{x}\right)\right| \\
& \leq\left(\psi^{-1}(1)+1\right) \gamma(|x-\bar{x}|) .
\end{aligned}
$$

Now, the continuity of $\gamma$ at 0 and the equality $\gamma(0)=0$, imply that $h$ is continuous with respect to the second variable.

Note that in Theorem 2.1 the function $\gamma:[0, \infty) \longrightarrow[0, \infty)$ is completely arbitrary.

As an immediate corollary of Theorem 2.1 and Remark 2.2 we obtain the following:

Theorem 2.3. Let $I \subset \mathbb{R}$ be an open interval, $\varphi, \psi \in \mathcal{F},\left(X,|\cdot|_{X}\right)$ and $\left(Y,|\cdot|_{Y}\right)$ be real Banach spaces, $C \subset X$ be a convex set such that int $C \neq \emptyset$ and $h$ : $I \times C \longrightarrow Y$. If the composition operator $H: C^{I} \longrightarrow Y^{I}$ generated by $h$, maps $B V_{\varphi}(I, C)$ into $B V_{\psi}(I, Y)$ and there exists a function $\gamma:[0, \infty) \longrightarrow[0, \infty)$ right continuous at 0 with $\gamma(0)=0$, such that

$$
\left\|H\left(f_{1}\right)-H\left(f_{2}\right)\right\|_{\psi} \leq \gamma\left(\left\|f_{1}-f_{2}\right\|_{\varphi}\right), \quad f_{1}, f_{2} \in B V_{\varphi}(I, C),
$$

then

$$
\begin{aligned}
& h^{-}(t, x)=a^{-}(t) x+b^{-}(t), \quad t \in I, x \in C, \\
& h^{+}(t, x)=a^{+}(t) x+b^{+}(t), \quad t \in I, x \in C,
\end{aligned}
$$

where $a^{-}, a^{+}: I \longrightarrow \mathcal{L}(X, Y)$, and $b^{-}, b^{+} \in B V_{\psi}(I, Y)$.

Remark 2.4. If $\gamma(t)=k t$ for some $k \geq 0$, then inequality (5) becomes the classical Lipschitz condition.

\section{Uniformly bounded composition operator}

Definition 3.1 ([9, Def. 1]). Let $X$ and $Y$ be two metric (or normed) spaces. We say that a mapping $H: X \longrightarrow Y$ is uniformly bounded if, for any $t>0$ there is a nonnegative real number $\gamma(t)$ such that for any nonempty set $B \subset X$ we have

$$
\operatorname{diam} B \leq t \quad \Longrightarrow \quad \operatorname{diam} H(B) \leq \gamma(t) .
$$

Remark 3.2. Obviously, every uniformly continuous operator or Lipschitzian operator is uniformly bounded. Note that, under the assumptions of this definition, every bounded operator is uniformly bounded.

The main result of this paper reads as follows.

Theorem 3.3. Let $I \subset \mathbb{R}$ be an arbitrary interval, $\varphi, \psi \in \mathcal{F},\left(X,|\cdot|_{X}\right)$ and $\left(Y,|\cdot|_{Y}\right)$ be real Banach spaces, and $C \subset X$ be a convex set such that int $C \neq \emptyset$. Suppose that the function $h: I \times C \longrightarrow Y$ is continuous with respect to the 
second variable. If the composition operator $H: C^{I} \longrightarrow Y^{I}$ generated by $h$, maps $B V_{\varphi}(I, C)$ into $B V_{\psi}(I, Y)$ and is uniformly bounded, then

(i) there exist $a^{-}: I^{-} \longrightarrow \mathcal{L}(X, Y)$ and $b^{-} \in B V_{\psi}\left(I^{-}, Y\right)$ such that

$$
h^{-}(t, x)=a^{-}(t) x+b^{-}(t), \quad t \in I^{-}, x \in C,
$$

(ii) there exist $a^{+}: I^{+} \longrightarrow \mathcal{L}(X, Y)$ and $b^{+} \in B V_{\psi}\left(I^{+}, Y\right)$ such that

$$
h^{+}(t, x)=a^{+}(t) x+b^{+}(t), \quad t \in I^{+}, x \in C .
$$

Proof. Take any $t \geq 0$ and arbitrary $f_{1}, f_{2} \in B V_{\varphi}(I, C)$ such that

$$
\left\|f_{1}-f_{2}\right\|_{\varphi} \leq t \text {. }
$$

Since $\operatorname{diam}\left\{f_{1}, f_{2}\right\} \leq t$, by the uniform boundedness of $H$, we have

$$
\operatorname{diam} H\left(\left\{f_{1}, f_{2}\right\}\right) \leq \gamma(t),
$$

that is

$$
\left\|H\left(f_{1}\right)-H\left(f_{2}\right)\right\|_{\psi}=\operatorname{diam} H\left(\left\{f_{1}, f_{2}\right\}\right) \leq \gamma\left(\left\|f_{1}-f_{2}\right\|_{\varphi}\right),
$$

and the result follows from Theorem 2.1.

Remark 3.4. If the function $\gamma:[0, \infty) \longrightarrow[0, \infty)$ in the Definition 3.1 is right continuous at 0 and $\gamma(0)=0$ (or if only $\gamma\left(0^{+}\right)=0$ ), then, clearly the uniform boundedness of the involved operator reduces to its uniform continuity.

It follows that Theorem 3.3 improves the result of [3, Th. 3.1] where $H$ is assumed to be uniformly continuous.

Consider the following:

Definition 3.5 ([9, Def. 2]). Let $X$ and $Y$ be two metric (or normed) spaces. We say that a mapping $H: X \longrightarrow Y$ is equidistantly uniformly bounded if, for any $t>0$ there is a nonnegative real number $\gamma(t)$ such that all $u, v \in B \subset X$,

$$
\operatorname{diam}\{u, v\}=t \quad \Longrightarrow \quad \operatorname{diam}\{H(u), H(v)\} \leq \gamma(t) .
$$

Of course, the equidistant uniform boundedness is a weaker condition than the uniform boundedness. The following result follows from Theorem 2.1.

Theorem 3.6. Let $I \subset \mathbb{R}$ be an arbitrary interval, $\varphi, \psi \in \mathcal{F},\left(X,|\cdot|_{X}\right)$ and $\left(Y,|\cdot|_{Y}\right)$ be real Banach spaces, and $C \subset X$ be a convex set such that int $C \neq \emptyset$. Suppose that the function $h: I \times C \longrightarrow Y$ is continuous with respect to the second variable. If the composition operator $H: C^{I} \longrightarrow Y^{I}$ generated by $h$, maps $B V_{\varphi}(I, C)$ into $B V_{\psi}(I, Y)$ and is equidistantly uniformly bounded, then

(i) there exist $a^{-}: I^{-} \longrightarrow \mathcal{L}(X, Y)$ and $b^{-} \in B V_{\psi}\left(I^{-}, Y\right)$ such that

$$
h^{-}(t, x)=a^{-}(t) x+b^{-}(t), \quad t \in I^{-}, x \in C,
$$

(ii) there exist $a^{+}: I^{+} \longrightarrow \mathcal{L}(X, Y)$ and $b^{+} \in B V_{\psi}\left(I^{+}, Y\right)$ such that

$$
h^{+}(t, x)=a^{+}(t) x+b^{+}(t), \quad t \in I^{+}, x \in C .
$$




\section{References}

[1] J. Appell and P. P. Zabrejko, Nonlinear Superposition Operator, Cambridge University Press, New York, 1990.

[2] V. V. Chistyakov, Mappings of generalized variation and composition operators, J. Math. Sci. (New York) 110 (2002), no. 2, 2455-2466.

[3] J. A. Guerrero, H. Leiva, J. Matkowski, and N. Merentes, Uniformly continuous composition operators in the space of bounded $\phi$-variation functions, Nonlinear Anal. 72 (2010), no. 6, 3119-3123.

[4] M. Kuczma, An Introduction to the Theory of Functional Equations and Inequalities, Polish Scientific Editors and Silesian University, Warszawa-Kraków-Katowice, 1985.

[5] K. Lichawski, J. Matkowski, and J. Miś, Locally defined operators in the space of differentiable functions, Bull. Polish Acad. Sci. Math. 37 (1989), no. 1-6, 315-325.

[6] W. A. Luxemburg, Banach Function Spaces, Ph.D. thesis, Technische Hogeschool te Delft, Netherlands, 1955.

[7] L. Maligranda and W. Orlicz, On some properties of functions of generalized variation, Monatsh. Math. 104 (1987), no. 1, 53-65.

[8] J. Matkowski, Functional equations and Nemytskii operators, Funkcial. Ekvac. 25 (1982), no. 2 127-132.

[9] Uniformly bounded composition operators between general Lipschitz function normed spaces, Topol. Methods Nonlinear Anal. 38 (2011), no. 2, 395-406.

[10] Uniformly continuous superposition operators in the spaces of bounded variation functions, Math. Nachr. 283 (2010), no. 7, 1060-1064.

[11] J. Matkowski and J. Miś, On a characterization of Lipschitzian operators of substitution in the space $B V\langle a, b\rangle$, Math. Nachr. 117 (1984), 155-159.

[12] J. Musielak and W. Orlicz, On generalized variations. I, Studia Math. 18 (1959), 11-41.

[13] H. Nakano, Modulared Semi-Ordered Linear Spaces, Tokyo, 1950.

[14] W. Orlicz, A note on modular spaces. I, Bull. Acad. Polon. Sci. Ser. Sci. Math. Astronom. Phys. 9 (1961), 157-162.

[15] N. Wiener, The quadratic variation of function and its Fourier coefficients, Massachusett J. Math. 3 (1924), 72-94.

[16] M. Wróbel, On functions of bounded $n$-th variation, Ann. Math. Sil. No. 15 (2001), 79-86.

[17] _ Lichawski-Matkowski-Miś theorem of locally defined operators for functions of several variables, Ann. Acad. Pedagog. Crac. Studia Math. 7 (2008), 15-22.

[18] Locally defined operators and a partial solution of a conjecture, Nonlinear Anal. 72 (2010), no. 1, 495-506.

[19] Representation theorem for local operators in the space of continuous and monotone functions, J. Math. Anal. Appl. 372 (2010), no. 1, 45-54.

[20] L Locally defined operators in Hölder's spaces, Nonlinear Anal. 74 (2011), no. 1, 317-323.

[21] L. C. Young, Sur une généralisation de la notion de variation de puissance p-ieme bornée au sens de N. Wiener, et sur la convergence des séries de Fourier, C. R. Acad. Sci. 204 (1937), no. 7, 470-472.

Dorota GŁazowska

FACUlty OF MAThEMATics

Computer Science And Econometrics

UNIVERSITY OF ZielonA GóRA

Zielona Góra, Poland

E-mail address: D.Glazowska@wmie.uz.zgora.pl 
José Atilio Guerrero

Departamento de Matemáticas y Física

Universidad NaCional Experimental del Táchira

San CRistóbal-Venezuela

E-mail address: jaguerrero4@gmail.com

JANUSZ MATKOWSKI

Institute OF MATHEMATiCS

University of Zielona Góra

Zielona Góra, Poland

E-mail address: J.Matkowski@wmie.uz.zgora.pl

Nelson Merentes

Escuela de Matemáticas

Universidad Central de Venezuela

Caracas-Venezuela

E-mail address: nmer@ciens.ucv.ve 